\title{
PSICOLOGÍA COMUNITARIA Y POLÍTICAS SOCIALES: TENSIONES EN EL QUEHACER DE LOS-AS PSICOLOGOS-AS COMUNITARIOS CHILENOS
}

\author{
Luis Opazo \\ Héctor Berroeta ${ }^{1}$ \\ Abel Guerra \\ Universidad de Valparaiso, Chile
}

\begin{abstract}
RESUMEN
Desde la década del 90 en Chile, las intervenciones en Psicología Comunitaria se han desarrollado principalmente dentro del marco simbólico, teórico y de recursos de las políticas sociales (Alfaro, 2012). Esta relación se ha caracterizado por una tensión entre los modelos de políticas públicas neoliberales y las dimensiones ético-políticas de la Psicología Comunitaria. En esta investigación se analiza el contenido de los relatos que construyen profesionales que trabajan en programas de políticas sociales en Chile, sobre las tensiones que se producen entre su quehacer, las políticas sociales y los principios de la Psicología Comunitaria. Se entrevista a diez psicólogos-as comunitarias-os, que tienen entre 2 a 26 años de experiencia trabajando en instituciones que ejecutan políticas sociales. Se reporta que la tensión en el quehacer profesional en la política pública se expresa en el grado de autonomía que otorgan los espacios de trabajo, la disputa profesional de su quehacer en la política pública y las consecuencias personales producto de las tensiones profesionales en sus espacios laborales.
\end{abstract}

\section{Palabras claves}

políticas sociales, Psicología Comunitaria, quehacer profesional

\begin{abstract}
Since the 1990s in Chile, interventions in community psychology have been developed mainly within the symbolic, theoretical and resources framework of social policies (Alfaro, 2012). This relationship has been characterized by a tension between neoliberal public policy models and the ethical-political dimensions of Community Psychology. This research analyzes the content of the stories that professionals who work in social policy programs in Chile build about the tensions that arise between their work, social policies and the principles of Community Psychology. We interviewed ten community psychologists, who have between 2 and 26 years of experience working in institutions that execute social policies. It is reported that the tension in professional work in public policy is expressed in the degree of autonomy granted by the work spaces, the professional dispute of their work in public policy and the personal consequences of professional tensions.
\end{abstract}

Keywords

social policies, Community Psychology, professional work

Correspondencia para este articulo debe ser referido al segundo autor en el correo hector.berroeta@uv.cl 


\section{COMMUNITY PSYCHOLOGY AND SOCIAL POLICIES: TENSIONS IN THE WORK OF CHILEAN COMMUNITY} PSYCHOLOGISTS

Los llamados gobiernos de la transición que tuvieron lugar en Chile a partir de los años 90, luego de 17 años de dictadura militar, impulsaron una serie de transformaciones políticas y económicas que tuvieron como consecuencia una reorientación estratégica de las políticas sociales, cuyo nuevo objetivo fue conservar el crecimiento que exhibió el modelo económico a mediado de los años 80, introduciendo acciones redistributivas para revertir las principales fuentes de inequidad que generó en la sociedad chilena (Alfaro \& Zambrano, 2009; De La Maza, 2004; Raczynski \& Serrano, 2005). Este sistema de focalización se complejizó progresivamente, de forma tal que el término "grupos vulnerables" comenzó a emplearse para incluir nuevos segmentos sociales prioritarios, a saber, los-as jóvenes, los-as ancianos-as, los-as niños-as, los-as pueblos originarios, entre otros. Al mismo tiempo, se crean un conjunto de instituciones para abordar problemas que emergen en estos grupos, tales como el consumo problemático de drogas, la violencia intrafamiliar y la reinserción social de niños, niñas y adolescentes (Alfaro \& Zambrano, 2009).

En este contexto de creciente oferta programática, gran parte de las prácticas de intervención de la Psicología Comunitaria en Chile se ha desarrollado principalmente dentro del marco técnico, material y simbólico de las políticas sociales (Alfaro, 2012). Esta forma de vinculación podría representar una oportunidad para favorecer el cambio social, bajo el supuesto de que la incidencia de la Psicología Comunitaria mejoraría el diseño de las políticas sociales, haciéndolas más concordantes con los requerimientos de las comunidades (Shinn, 2007; Alfaro \& Zambrano, 2009). Sin embargo, una serie de investigaciones constatan que aún cuando puedan existir puntos de encuentro, las políticas sociales implican una serie de riesgos y tensiones para la Psicología Comunitaria (Alfaro \& Martín, 2015; Alfaro \& Zambrano, 2009; Nelson, Lavoie \& Mitchell, 2007; Nelson, 2013; Rodríguez, 2009; 2012; Serrano-García, 2013).

Particularmente, determinados modelos de políticas públicas, caracterizadas por formas asistenciales, paliativas, compensatorias o centradas en el déficit, propias de concepciones subsidiarias del Estado, podrían amenazar el carácter crítico y transformador de la Psicología Comunitaria (PC), limitándose también la promoción de procesos abajo-arriba propios del discurso disciplinar (Alfaro, 2013). En cuanto a la incidencia de la Psicología Comunitaria en las políticas públicas chilenas, el estudio de Reyes, Olivares, Berroeta \& Winkler (2015) constata un aporte marginal considerando el grado de desarrollo disciplinar en el país, denunciando escasa consideración de los principios y prácticas de la Psicología Comunitaria en la mayoría de los programas comunitarios analizados, los que incorporan sólo aspectos técnico-metodológicos.

Esta relación, compleja y de larga data en el diseño y ejecución de programas sociales (Reyes, Olivares, Berroeta \& Winkler, 2015), le ha ofrecido a la PC "una base material para su desarrollo como campo de especialización profesional de la Psicología facilitando su desarrollo académico dentro de la institucionalidad universitaria nacional" (Alfaro \& Zambrano, 2009, p. 280).

\section{Tensiones entre el quehacer profesional de la Psicología Comunitaria y las políticas sociales}

En el desarrollo disciplinar se evidencia una relación complicada entre el quehacer de la Psicología Comunitaria y el marco institucional de las políticas públicas, en tanto a los objetivos transformadores que orientan el cambio social de la disciplina, se le contraponen los objetivos que persiguen los Estados (Serrano-García, 2013; Phillips, 2000; Perkins, 1995; Nelson, 2013). Alfaro (2012) plantea que habría una clara tensión y desencuentro entre las orientaciones de los programas de políticas sociales y las orienteaicones de la Psicología Comunitaria que se expresa en los planos de los valóres y la ética, las estrategías de intervención y los modelos de referenicas que sustenta el problema. 
Tensiones entre PC y políticas sociales en Chile. La relación entre PC y políticas sociales en Chile se ha traducido en un enfoque individual para el abordaje de las problemáticas sociales (Alfaro, 2000; 2007; Berroeta, 2011; Berroeta et.al, 2012), el que, para Berroeta (2011), se ha conceptualizado como "enfoque psicosocial" y, operativamente, como "intervención psicosocial", asignando "poco valor a las condicionantes sociales, a los recursos comunitarios y a la participación ciudadana" (p. 41), considerándolo incompatible con la transformación social. Este enfoque individual, consolidado en los 2000, relevaría a un segundo plano las iniciativas territoriales y estaría relacionado con la distancia que adoptó la disciplina respecto de sus propios principios transformadores a partir del fuerte proceso de institucionalización experimentado (Berroeta y cols. 2012), constatandose que "el paradigma de la psicología comunitaria tal cual lo conocemos, no tiene cabida explícita ni fluida en la actual estructura normativa de la política social chilena." (párr. 55), a pesar de su interés y preocupación por las políticas sociales (Alfaro, 2012b).

No obstante en la actualidad es posible distinguir algunos elementos promovidos por la PC en las políticas sociales chilenas, como la incorporación de un enfoque participativo, la consideración del sentido de comunidad, la acción territorial y el fortalecimiento comunitario, como ejes programáticos de la acción en algunas de las propuestas de intervención (Reyes et. al., 2015), elementos que pueden servir de sustento para estrategias futuras que avancen en los componentes comunitario y participativo (Zambrano \& Bustamante, 2012).

Estas particularidades, que pueden ser leídas como tensiones, se manifiestan en el nivel del quehacer profesional, a través de un rol difuso, una insuficiencia en la formación profesional, poca expereciencia en el área y alta rotación en los programas sociales en los cuales se integran (Winkler y colb. 2010: 2015). Sumado a las escasas posibilidades de aplicar los marcos ético-políticos de la disciplina, debido a las exigencias institucionales de sus prácticas (Berroeta, 2011), esto provoca un marcado descontento en los-as profesionales.

La investigación de Winkler, Alvear, Olivares y Pasmanik (2010) referente a las prácticas profesionales en el ámbito comunitario, da cuenta de la dificultad de diseñar y ejecutar prácticas comunitarias en las políticas públicas, donde las acciones desplegadas son evaluadas frecuentemente desde el resultado y en función de indicadores cuantitativos. Asimismo, se destaca la falta de autonomía, entendida como el escaso control profesional en las intervenciones y sus objetivos, como un factor que interfiere y tensiona el desarrollo de este quehacer, delimitando sus acciones y alcances en el terreno comunitario. No obstante, existen algunas acciones que, si bien operan sobre un nivel individual, se orientan hacia la transformación y son ejecutadas con pequeños márgenes de autonomía (Berroeta, 2014), muchas veces dispuestos desde las mismas políticas sociales (Berroeta, Hatibovic, \& Asún, 2012).

La investigación que aquí se reporta tuvo como objetivo analizar el contenido de los relatos que construyen profesionales que trabajan en programas de políticas sociales en Chile, sobre las tensiones que se producen entre su quehacer, las políticas sociales y los principios de la Psicología Comunitaria.

\section{Participantes y procedimiento}

\section{Método}

En esta investigación participaron quince profesionales del área de la Psicología pertenecientes a las regiones de Valparaíso y Metropolitana de Chile, con al menos dos años de experiencia de trabajo comunitario en programas de políticas sociales. Dichos participantes fueron seleccionados mediante la técnica bola de nieve (Ruiz, 2012) y contactados vía correo electrónico. Para resguardar los principios éticos de la investigación se aplicó un consentimiento informado sobre los objetivos y límites de la investigación. A continuación se da cuenta de los-as participantes, la institución a la que pertenecen y los años de experiencia. 
Tabla $\mathbf{N}^{\circ} \mathbf{1}$

Caracterización de participantes.

\begin{tabular}{|l|l|l|}
\hline Participante & $\begin{array}{l}\text { Institución o programa de política pública en la cual se } \\
\text { desempeña }\end{array}$ & $\begin{array}{l}\text { Experiencia } \\
\text { laboral }\end{array}$ \\
\hline E1 & $\begin{array}{l}\text { Corporación Servicio Paz y Justicia - Servicio Nacional de } \\
\text { Menores }\end{array}$ & 21 años \\
\hline E2 & $\begin{array}{l}\text { Servicio en beneficio de la comunidad - Servicio Nacional } \\
\text { de Menores }\end{array}$ & 6 años \\
\hline E3 & $\begin{array}{l}\text { Programa de Acompañamiento y Acceso Efectivo a la } \\
\text { Educación Superior - Ministerio de Educación. }\end{array}$ & 2 años \\
\hline E4 & $\begin{array}{l}\text { Programa de Acompañamiento y Acceso Efectivo a la } \\
\text { Educación Superior - Ministerio de Educación. }\end{array}$ & 3 años \\
\hline E5 & Techo & 3 años \\
\hline E6 & $\begin{array}{l}\text { Programas Especializados en Maltrato y Abuso Sexual } \\
\text { Grave - Servicio Nacional de Menores }\end{array}$ & 4 años \\
\hline E7 & Centro Comunitario de Salud Familiar. & 8 años \\
\hline E8 & $\begin{array}{l}\text { Servicio Nacional de Menores - Fondo de Solidaridad e } \\
\text { Inversión Social }\end{array}$ & 26 años \\
\hline E9 & $\begin{array}{l}\text { Corporación Servicio Paz y Justicia - Servicio Nacional de } \\
\text { Menores }\end{array}$ & 8 años \\
\hline E10 & Inclusión Social Calle - Hogar de Cristo & 4 años \\
\hline
\end{tabular}

\section{Técnica de producción de datos}

La técnica de producción de información utilizada fue la entrevista semi-estructurada individual, instrumento que permitió abordar la experiencia de psicólogas-os en sus espacios de trabajo, posibilitando determinar el quehacer de quienes participan en los programas de políticas sociales. En torno a tres dimensiones: a) características de la institución o programa en el cual trabajan; b) quehacer profesional y c) relación de la Psicología Comunitaria con su quehacer.

\section{Técnica de análisis: análisis temático}

La información producida en las entrevistas semi-estructuradas fue analizada mediante análisis temático (Braun \& Clarke, 2006; Willig, 2013). Un tema refiere a una configuración particular y reconocible de significados que co-ocurren significativa y sistemáticamente (Willig, 2013, p. 181). Para Braun y Clarke (2006, p. 82), los temas capturan aspectos importantes de los datos en relación con la pregunta de investigación, representando en algún nivel los patrones de respuesta o de significados en un conjunto de datos. El análisis temático produce conocimiento en forma de temas, hechos a partir de códigos descriptivos, que capturan y hacen sentido de los significados que caracterizan el fenómeno a investigar (Braun \& Clarke, 2006). Se reportan significados semánticos de los datos, explícitamente observables; o significados latentes, supuestos e ideas que están detrás de lo que se afirma de manera explícita (Braun \& Clarke, 2006; 2012; Willig, 2013).

\section{Resultados}

Los relatos de los participante fueron ordanados entorno a un tema central que articula las narraciones y que denominamos "tensiones en el quehacer profesional en la política pública" y un tema subordinado que describe las lecturas y posciones que las y los profesionales asumen frente a la política pública, dentro de cual identificamos tres subtemas a) grados de autonomía; b) disputa 
profesional de su quehaber en las políticas públicas; y c) consecuencias personales que asume el-la profesional.

\section{Figura $\mathbf{N}^{\mathbf{0}} 1$}

Mapa temático

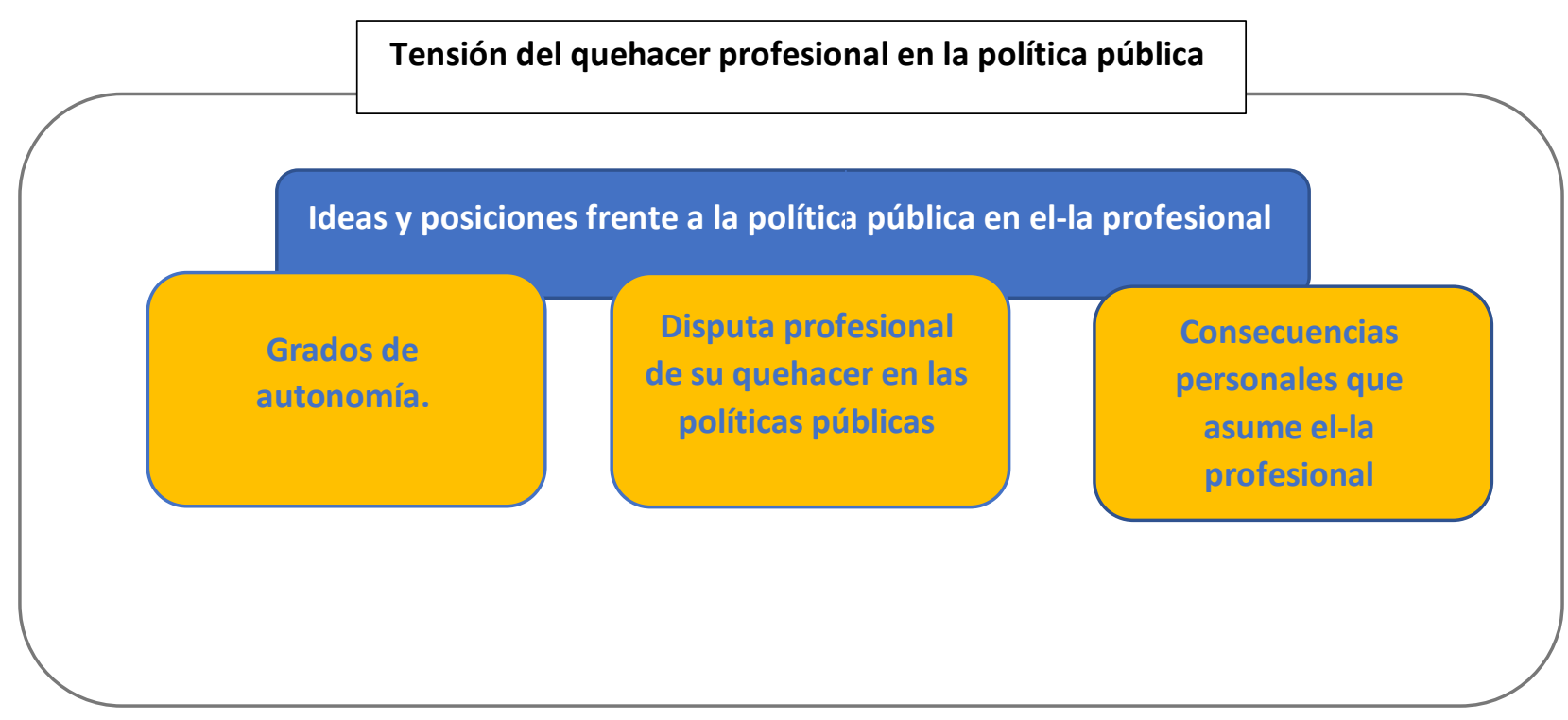

Tensión del quehacer profesional con las política públicas.

Los relatos de los-as psicólogos-as comunitarios-as describen dificultades para desarrollar su quehacer que se expresan como una "tensión" con la política pública, a nivel instucional, profesional y personal. Estas lecturas problematizadoras, al mismo tiempo, propician el reconocimiento de posibilidades de incidir en ellas, en consonancia con los valores de la PC (Montero, 2001; 2004; Prilleltensky, 2004) o sus propios sentidos del quehacer ético-político de la disciplina. De este modo, los-as profesionales asumen una serie de roles dirigidos a la modificación de la política pública (Bishop, Vicary, Browne \& Guardo, 2009; Nelson, 2013; Shinn, 2007).

Estas tensiones se caracterizan por limitaciones que supone el diseño de programas de intervención de corto plazo, lo que dificulta la implementación de procesos participativos (Lapalma \& De Lellis, 2012).

Tú empiezas a hacer un trabajo comunitario, la gente empieza a participar, empieza a venir la mamá, vienen los niños, toda la cosa, pero en algún momento qué pasa, se corta el proyecto, se corta la plata, y tú, lamentable, como profesional no puedes seguir trabajando voluntariamente porque tienes que trabajar, tienes que vivir (E8, Grupol).

Discontinuidad de los procesos de intervención, así como la dificultad de realizar un seguimiento pertinente de los resultados alcanzados.

Los problemas es la continuidad de los procesos que tú haces, que no los puedes seguir, porque tú cuando empoderas a una señora... (E8, Grupol).

Cuestionamiento a la propuesta de las políticas públicas que prioriza una intervencióna a nivel individual, por sobre las posibilidades del trabajo en un nivel colectivo-comunitario. 
Como programa de justicia juvenil de niños y niñas, cabros chicos' qué se yo, ¿no deberíamos instalar en la comunidad el tema de, por ejemplo, las detenciones ciudadanas? O sea, cómo...yo creo que eso sí es un trabajo comunitario, porque lo que tú haces es abrir un diálogo sobre un problema social ahí y entre colectivos, o sea, yo decía: ¿no deberíamos trabajar como, por ejemplo, con todos los programas sociales del área? (E2, Individual).

Una evaluación de las políticas públicas que tienden a ser autocomplacientes, dificultando evaluar los efectos del quehacer definidos por los programas; en los que se privilegia la consecución de metas (en términos cuantitativos) por sobre los procesos, generando una percepción de reducción de las situaciones y las personas a cifras, homologando los procesos interventivos a los de producción en serie.

Me cuesta, de verdad, decir hasta dónde esto tuvo que ver con la intervención y hasta dónde no, así como...y, por ejemplo, una cuestión súper heavys, que nosotros en el programa siempre problematizamos, hasta dónde...pero que, no, me estoy escapando igual, un poco, pero, hasta dónde las cosas tienen que ver, por ejemplo, con el vínculo que estableciste con alguien, con la familia, con la comunidad y con la técnica que ocupaste, ¿cachai + No sé, por ejemplo, ese es otro rollos como bien, bien heavy, porque uno trabaja con personas po', y al final como que...y yo creo que los comunitarios eso es lo que siempre están teniendo ahí, que son personas, que son personas, que no es un número, que no es una maqueta, que no es un trabajo en serie de algo y etcétera (E2, Individual).

Finalmente se da cuenta de una alta estruturación e insensibilidad en el modo en que se ejecuta el control normativo de las política pública ante situaciones que pudiesen ser emocional y eticamente complejas de abordar para las-os interventoras-es.

Como que el otro día tenía una cabra en el hospital y le tuvimos que ir a comprar toallas y, no sé po', confores, y como que yo después le decía al supervisor: ¿De verdad querías que a la mamá, llorando, porque la cabra estaba en la calle, le pidiera una firma? O sea, sorry po', hueón, ya me la guardo yo para el bolsillo mío, pero no voy a hacer esa inhumanidad (E2, Individual).

Ideas y posiciones frente a la política pública en el-la profesion. La tensión entre el quehacer y la política se refleja en el relato de los-as profesionales sobre las acciones que desarrollan en torno a las políticas públicas, discurso marcado por un constante cuestionamiento a las posibilidades de realizar prácticas transformadoras en este marco, visión fundamentada en la incongruencia de la acción con los desarrollos teóricos y valóricos de la PC.

Esta noción es descrita como dificultades con el abordaje de los problemas sobre los cuales se interviene, dado que estos son el resultados de condiciones estructurales, que incluso se ven reforzados por las políticas públicas, en tanto reproducen dinámicas de desigualdad y exclusión. Los relatos expresan una posición crítica de los-as profesioanles con los sentidos que despliegan las políticas públicas a través de los programas:

Si, partiendo del diagnóstico de que estamos en un país sumamente desigual, uno de los más desiguales del mundo, y que no es por casualidad, jugado a largo de los años se ha perpetuado un sistema que avala y potencia la desigualdad (E10, Grupo 2).

\footnotetext{
Nominación coloquial que reciben los-as niños-as.

Expresión que da cuenta que un hecho tiene un alto grado de impacto.

Expresión coloquial usada para preguntar al interlocutor si entendió una idea expuesta.

Expresión que da cuenta de otro punto a considerar en su argumento.
} 
La gente no tiene tiempo, los adultos no tienen tiempo para hacer sus cosas y como nunca tienen tiempo para hacer sus cosas, cuando lo tienen, no saben qué hacer con ellos, y como que eso genera una hueá ${ }^{\sigma}$ súper rara, o sea, a ver, me tengo que citar desde la pega que hago, pero cuando yo veo a los cabros tirados, tirados así, solos, hueón, tirados como así, como...valiéndoselas por las de ellos mismos a los catorce años, ¿cachai? y, ¿dónde está la mamá? Resulta que la mamá, de los trece años que trabaja veinte horas diarias y tiene que dejar al cabro encerrado no más po', y como que lo tiene que dejar encerrado para que el cabro chico no se pierda y eso es vulneración de derechos, chucha. Y, entonces, ¿cómo le trae comida? ¿Cachai? Es tiempo, si es de...sorry, hueón, yo de verdad que en estas discusiones siempre yo, así, como que quedo como la mala de la película, porque de verdad yo creo que estas cuestiones derivan de problemas estructurales, y mientras esos no se tomen, no se topen, hueón, no, no lo entiendo. Al menos voy a ponerlo desde eso [J: Un psicólogo comunitario del Ministerio po, hueón...]. Entonces, como que creo que el problema de las comunidades, hoy día, son los tiempos, son los accesos que tienen a lo que es la vida, y lo que es la vida desde como las necesidades básicas hasta...no sé po', hueón (E2, Grupo 1).

Falencias en el diseño de los programas en que trabajan, que originan una serie de dificultades para su operacionalización. En general las interpelaciones refieren a que las indicaciones y procedimientos resultan insuficientes para las situaciones complejas que enfrentan los profesionales, se describen como causas de estas falencias, la falta de evidencia técnica que respalde la política pública.

La política pública son dos personas sentadas en un escritorio, que se les ocurre una genialidad y generan todo un proceso para ejecutarlo, pero sin ninguna evidencia, $o$ sea, a nosotros (...): no sabemos si hay programas de orientación, no sabemos cuáles son los mejores programas para UTP, por ejemplo, no sabemos cuáles son las bases o establecer como son la nueva subjetividad del joven, y eso te lo debería dar el programa, eso no debería estar ejecutado por ti como profesional, si tú generas una política pública, tiene que venir dotada de esta evidencia, y no porque alguien te dijo que era importante la inclusión, casi desde el sentido común, y no es que no lo comparta, pero si, cómo eso tú lo vas ejecutando, y tener a la mano también otras experiencias que te marquen el camino (E3, Individual).

Los-as profesionales refieren una falta de coherencia en distintos niveles de la politica pública,programáticos, metodológicos, políticos y éticos. La cual se expresa, incluso, en una desarticulación entre distintos programas de políticas públicas.

Yo genero una acción muy desde ciertos sentidos, pero viene tu compañero que trabaja con los mismos actores, pero con una visión totalmente diferente, generas esta incoherencia, que no es lo que tú, o sea, o por lo menos la PC pretende trabajar (E3, Individual).

Lo que pasa hoy día es, generas un programa como el Programa de acceso y acomañamiento efectivo a la educación superior (PACE), lo insertas en una comunidad educativa, que están sobre intervenidas, e intervenidas de lógicas muy diferentes, o sea,

- Expresión que refiera a una cosa y/o situación.

- Unidad Técnica Pedagógica, es el departamento que en los establecimientos educativos básicos y medios que está a cargo del trabajo técnico pedagógico. 
Fundación Chile, la lógica de Fundación Chile va a ser totalmente diferente de la que viene de una universidad como la Valparaíso, insisto, incluso en el mismo PACE, la visión programática que va a tener la Santa María, o la Católica, va a ser muy diferente a la visión programática de la Valparaíso o la UPLA (Univerisdad de Playa Ancha), y eso te va generando una inconsistencia desde lo ético, desde lo político, una inconsistencia de como construyen el problema, una inconsistencia en las metodologías que utilizan (E3, Individual).

Precariedad en las condiciones para implementar las políticas públicas, falta de recursos para ejecutar las intervenciones, pocas posibilidades de capacitación, pocas horas destinadas a intervenciones clínicas y comunitarias, que afectan la calidad de las intervenciones que se realizan.

El comunitario tiene que trabajar con esa precariedad po', y casi siento que, en cierta medida, no solamente el comunitario, sino que, en general, los psicólogos, estamos entrando en un lugar donde si no tienes dinero no te puedes capacitar, y no sé si eso sea así en todas partes del mundo, y no sé si eso tenga que ser así también, en Chile, para siempre (E7, Individual).

... porque quizá para mi hubiera sido mucho más fácil seguir haciendo intervención individual, pero el beneficio, quizá el logro del objetivo no lo hubiera logrado nunca, pensando también que es súper acotada la intervención, son 18 meses y ahora para el próximo año van a ser 12, cada vez es menos el tiempo de intervención, porque lo que pide la política pública finalmente es que le den la atención al niño ni siquiera al adulto y cuando uno, cuando los niños llegan, porque los niños no llegan solos llegan con un adulto, el adulto a veces tiene una historia de daño que es mucho más grande que la del niño... (E1, Individual)

Por último se destaca que la ejecución de los programas demanda múltiples roles profesionales que no se condicen con las formación académica recibida, ni con la remuneración pactada.

A ella le carga cuando empiezan a puntualizar a SENAME (Servicio Nacional del Menor) de ciertas cosas, pero la loca me dice (...) qué posgrado, que posgrado, qué postítulo, qué posgrado en Harvard, la hueá que sea, te dice y te enseña como tú abordas una crisis de una cabra, ¿quién? Tendrías que ser médico, paramédico, bombera, psicóloga, profe, no sé, ¿cachai? Tendrías que ser todo y tener todo súper integrado para abordar eso, y ahí está la falencia, el corte, ella lo centra mucho más en cómo es la preparación que tienes, esté el que esté ahí va a quedar la caga, y yo le digo: Ya, sí, esté el que esté ahí va a quedar la caga, pero alguien diseño quienes iban a estar ahí (E2, Individual).

Estas características determinan el tipo de relación que los-as profesionales establecen con la institución y el modo específico en que se despliega su quehacer profesional. A continuación se presentan tres subtemas que expresan las dimensiones que están involucradas en este despliegue específico.

\section{Subtema 1: Grados de autonomía}

Este subtema da cuenta de las condiciones de posibilidad definidas por los programas de políticas sociales mediante las cuales los-as profesionales intentan, en su quehacer, introducir enfoques y desplegar acciones alternativas a las que están presentes en las instituciones en las que se desempeñan laboralmente. 
Los grados de autonomía en la acción profesional son diversos y amplios, se describe que existen margenes de libertad para que los-las profesionales definan las acciones específicas de su quehacer, especiamente en el modo en que se diseña y aplica herramientas metodológicas en el espacio laboral, esto aun cuando existe una programación predefinidas de los productos por parte de la institución en la cual se desempeñan:

Yo creo que había un diseño, una maqueta, por así decirlo, muy clara de lo que habia que hacer, con momentos hitos, predefinida, con apertura a la creatividad también en la acción que se permitía, digamos, uno podía generar algún tipo de estrategia innovadora, algún tipo de proceso de registro, ya sea desde el formato audiovisual, el trabajo con los niños, con los jóvenes, pero el tronco, digamos, estaba muy bien parado para pensarse ahí uno como profesional (E9, Individual).

Las-os profesionales aprovechan estos grados de autonomía para incorporar concepciones o modelos teórico-interventivos vinculados a la Psicología Comunitaria en su quehacer profesional.

Ese dispositivo generó una publicación, y esa publicación llegó a mis manos cuando tuve que hacerme responsable de mi trabajo o responsable de mi trabajo en una intervención postincendio, en un consultorio que había sido consumido por llamas. $Y$, en términos prácticos, parecía más o menos plausible tratar de replicar ese modelo en ese espacio, y se hace la propuesta, y este trabajo se presentó en la Universidad de Chile, a propósito de la Escuela de Salud Pública, después del incendio (E7).

Sin embargo, según el reporte de los participantes, estas acciones no suelen modificar sustancialmente niveles fundamentales de las políticas sociales, tales como las disposiciones legislativas que definen los cursos de acción para los programas en los que trabajan los-las profesionales. Así lo reconoce una profesional, respecto a su participación en el Consejo Asesor de Infancia del gobierno de Michel Bachelet para incidir en las políticas de infancia:

Salió el informe, que era un informe bien potente a mi parecer, que pensaba más en lógicas locales, y ahí el lugar de la PC tenía mucha relevancia, muchas posibilidades, pero luego, por decisión política, pasando por el parlamento se redujo al Chile Crece Contigo (Programa del ministerio de la Salud), y por lo tanto no tuvo impacto en las políticas de infancia en general, por ejemplo, que ese consejo asesor debería haberlas tenido (E1).

Cuando existen grados reducidos de autonomía institucional, disminuye el margen de libertad de las profesionales para desplegar su quehacer, debiendo responder a las exigencias de las instituciones en las que se desempeñan:

Es una cuestión básica, si eres trabajador, hueón, en el consultorio yo lo vi, los locos tienen que tener unas metas de gente atendida por depresión, y a la gente que no tiene depresión le tienen que poner depresión, ¿cachai? (E2, Grupol).

Sin embargo, aún bajo restringidos márgenes de libertad, los-las profesionales advierten que existen espacios de autonomía que les permiten desplegar acciones con sentido transformador, especialmente en las relaciones cotidianas que establecen con los sujetos destinatarios de las instituciones de políticas públicas en las que trabajan:

Siento que hoy día hay pegas en la que está la gente, así como de interventores medios, están súper coartados, y lo que tú generas es una relación diferente con la persona, en donde puedes compartir sentidos comunes, qué sé yo, posiciones, qué se yo, aquí, allá. Pero tu objetivo de trabajo como tal, hueón, pasa por los locos que te están diciendo que tienes que hacer, los números que tienes que tener (E2, Grupol). 
Estas acciones revisten la forma de astucias, toda vez que las profesionales aprovechan estos espacios para desplegar principios y acciones vinculados a la PC, satisfaciendo, al mismo tiempo las exigencias institucionales

Es como también uno instrumentaliza los dispositivos con los que uno cuenta en el espacio ínfimo que es la intervención, yo creo que ahi es justamente cuando entrega posibilidades y si tenemos la capacidad para justamente incorporar en estos planes de intervención que son individuales algún elemento, por más pequeño que sea, desde lo comunitario (E6, Individual).

\section{Subtema 2. Disputa profesional de su quehacer en la Política Pública}

Este subtema da cuenta del modo en que se asume una posición ético-política en el quehacer, asociado a una consecuencia en la trayectoria biográfica e identitaria que es aparejada con una lectura situada de la disciplina.

Yo creo que ahí se adosan un elemento identitario, un elemento militante. La Psicología Comunitaria es una disciplina, y como tal se puede desprender en momentos y en contextos. Es una profesión con una lectura de la ciencia social particular, emerge y nace, por tanto, esa idea de pegártela y andar para todas partes, y sentir que eres inconsecuente, eso es de los años setenta, ¿cachai?, cuando había y se podía, cuando era legitima la dicotomía (E4, Grupo1).

"El TAF(Taller de Acción de Forestal) mismo y Epifanía(Ong), mira, ¿qué hicieron? fueron consecuentes con la educación popular y todo. ¿Y qué hicieron de SENAME? ipum!, marginado, ¿por qué? eso es lo que pasa. Me siento huérfano, porque ahí donde...es la consecuencia” (E8, Grupol)

Una posición posible es aquella que asume, aún a riesgo de perder el trabajo, que el profesional debe orientar sus prácticas hacia el desarrollo de procesos de fortalecimiento y disputa de derechos, en concordancia con los principios ético-políticos de la disciplina.

Por ejemplo, está la caga allá con la minera, "señora, ¿sabe qué?, vamos a hacer lo siguiente: tomémonos la calle y llamemos a televisión nacional”. Y quedó la grande, se tomaron la cancha, y ganaron todos y me echaron a mí, pero lograron hacer un camino nuevo, pusieron semáforos y todo el asunto. ¿Quién cumplió? Yo cumpli la misión de empoderarlos y la comunidad se organizó y la junta de vecinos ahora tiene caminos, semáforos, todo, claro, quedé sin pega yo, pero la comunidad creció y participó (E8, Grupo1).

Otras posición posible del-la profesional es aquella que se adapta a las circunstancias de trabajo en el marco institucional, de modo que el-la profesional, en el espacio de intervención, adopta posiciones acordes a las lecturas del contexto en el cual se sitúa. Establece como condición de la acción, una comunicación clara y transparente de las condiciones institucionales y los objetivos de la intervención.

También ser súper transparente en qué es lo que a ti te tiene ahí y qué no, o sea, cuando tú tienes una pega, ya: yo tengo una pega, tengo aquí unos objetivos, "mire, ¿sabe?, yo tengo que hacer esto y esto otro". Cuando yo voy porque es una acción social o política la que estoy haciendo, "sabe qué, yo vengo porque sí poh: yo creo que aquí en la población hay que trabajar y quiero trabajar por esto, por esto y esto otro con usted", pero es como una transparencia de plantear "sí, esto es lo que quiero hacer, ya: ¿le parece o no le parece? Démosle (E2, Grupo1). 
Desde esta segunda posición, reconocen nuevas condiciones para el trabajo comunitario, que distan de lo que ellos-as interpretan eran las condiciones sociohistóricas cuando emergió la psicología comunitaria, por tanto accionan desde otro lugar su quehacer.

Esa idea de pegártela y andar para todas partes, y sentir que eres inconsecuente, eso es de los años setenta, ¿cachai?, cuando había y se podía, cuando era legitima la dicotomía, pero en estos contextos más híbridos, muchos más complejos, mucha más multiplicidad de realidades, condiciones es muy difícil generar eso (...) Probablemente la sociedad también cambió po'. También la pretensión, es lo que, insisto, como que uno pretende siempre volver a configuraciones de años ochenta, cuando tenían sentido, cuando la población obrera tenía sentido, cuando las grandes urbes de trabajadores, cuando los cordones industriales...” (E4, Grupo1).

\section{Subtema 3: Consecuencias personales que asume el-la profesional}

Las tensiones que vivencian con la institucionalidad y las diferentes posiciones que asumen los-las profesionales para enfrentarlas, tiene efectos personales que caracterizan el modo en que el profesional vivencia el ámbito laboral .

Cuando los-as profesionales no estan dispuestos a renunciar o a flexibilizar el modo en que se despliega en las intervenciones los principios de la PC lleva al- a que estos-as profesionales se desempeñen bajo un permanente malestar.

"Yo creo que [en mi quehacer había] un espíritu de resiliencia, un espíritu no más, quizás como una expectativa de cambio bien inocente, pero digamos con este carácter de más resiliencia, así como de darle, darle, darle, hasta el punto de quemarte" (E9, Individual)

...o sea llega un momento determinado que tú te empiezas como a, puede haber una confusión ahí entre vinculo y enajenación, pero claro uno piensa que ese estar ahí, con ese equipo, con esos compañeros y compañeras, se transforma en una manera de ser también profesional, claro abusando un poco, yo creo, de uno mismo, de su tolerancia: “¿cuál es el límite?”, eso que era muy auto, era muy poco facilitador de tomar ciertas decisiones. Cuando yo pude identificar algunos momentos que estaba quemado, pero, aun así, ya fuera por la necesidad concreta de la subsistencia o la ambigüedad del escenario futuro o de la lectura que yo le estaba dando al escenario futuro, complicaba tomar una decisión concreta: vamos a ver, qué más hay, no hay más ofertas entonces te quedas aquí. Aun así, yo creo que, las expectativas de ser útil o de sentirse útil juegan bastante, sobre todo cuando encuentras equipos donde eso es reconocido colectivamente, no como un reconocimiento individualizado... (E3, Individual)

Las necesidades de la acción profesional requiere que esta se realice fuera de los horarios formales estabecidos por el contrato laboral, lo que genera en las-los profesionales la sensación de una sobredemanda y permanete precarización laboral.

“QQué hago yo? Parto para allá, sábado y domingo” (E8, Grupol)

"Pero cuando yo llegaba a la casa, y la señora no estaba, no me iba para la casa y le decía a la señora que no la encontré: iba a la hora de almuerzo, iba de noche o sábado y domingo" (E8, Grupol)

En síntesis, el analisis temático nos muestra que las y los profeionales despliegan su quehacer en un contexto de tensiones con las políticas publicas, las que son descritas en distintos niveles y 
dimensiones, tales como, discontinuidad de procesos, foco prioritario en lo individual, centrada en productos y no procesos, insensibiidad frente a situaciones complejas, etc. Aspectos que en su conjunto, condicionan las posibilidades de llevar a cabo una práctica consecuente con los pincipios etico-políticos de la psicología comunitaria. Esta dificultad para realizar practicas transformadoras tienen que ver con los sentidos de la política pública, su diseño e implmentación; características que se configuran como contexto para la práctica desde el cuál se reconocen grados de autonomía que se aprovechan para realizat acciones complementarias o alternativas a lo establecido formalmente por las instituciones. En este ejerecio los y las profesionales adoptan posiciones más o menos adaptatadas a las condiciones que propone la política púbica, a la vez que asumen consecuencias personales derivadas de su quehacer, como la inestabilidad laboral y el trabajo fuera de horarios. 


\section{Discusión y Conclusiones}

En concordancia con las conclusiones de Berroeta (2014), mientras el despliegue de tácticas en márgenes de autonomía reducidos posibilita que los-as profesionales aprovechen el espacio relacional con los-as destinatarios para el desarrollo de prácticas orientadas a la transformación; el despliegue de estrategias no suele incidir en los aspectos más fundamentales de las políticas sociales, más bien, los-as profesionales logran implementar modelos de intervención orientados desde la Psicología Comunitaria dentro de los programas en los cuales se desempeñan, tal como lo señalan las conclusiones de Reyes \& cols. (2015).

Por otra parte, la adopción de una posición militante supone que los-as profesionales conciban su quehacer como un compromiso ético y político que incluso logra permear dimensiones de su vida personal distintas al contexto laboral, tal como lo advierte Reyes (2007). Respecto de la adopción de la posición negociante, en coherencia con los planteamientos de Krause \& cols. (2011), los-as profesionales intentan dialogar con las políticas sociales, compatibilizando en ese ejercicio, las demandas de su trabajo con los principios y planteamientos de la Psicología Comunitaria.

En suma, podemos conlcuir que el quehacer de los-as profesionales que desarrollan acción comunitaria en programas de politicas públicas, se despliega de un modo complejo y diverso, adaptando de una manera situada sus prácticas según valoran los principios disciplinares, su pertinencia contextual en el quehacer específico y leen el margen de autonomía que les otorga la institucionalidad de las políticas públicas. 


\section{Referencias}

Alfaro, J. (2000). Discusiones en psicología comunitaria. Santiago, Chile: RIL Editores.

Alfaro, J. (2007). Políticas sociales como condición de posibilidad para el desarrollo de prácticas en Psicología Comunitaria. En J. Alfaro \& H. Berroeta (Eds.), Trayectorias de la Psicología Comunitaria en Chile: Prácticas y conceptos (pp. 43-72). Valparaíso, Chile: Universidad de Valparaíso.

Alfaro, J. (2012). Posibilidades y tensiones en la relación entre Psicología Comunitaria y políticas sociales. En J. Alfaro, A. Sánchez \& A. Zambrano (Comps.), Psicología Comunitaria y Políticas Sociales. Reflexiones y experiencias, (pp. 45- 75). Buenos Aires, Argentina: Paidós.

Alfaro, J. (2012b). Psicología Comunitaria y políticas sociales: Análisis del Programa Chile Solidario desde la óptica de la Psicología Comunitaria. Revista Latinoamericana de Psicología Social Ignacio Martín-Baró, 1(1), 154-172.

Alfaro, J. (2013). Psicología comunitaria y políticas sociales: estudio del campo técnico del psicólogo de la intervención social en el marco de los servicios sociales comunitarios españoles. (Tesis doctoral). Universidad de Girona, España. Recuperado de http://hdl.handle.net/10803/108340

Alfaro, J., \& Martín, M. P. (2015). Proceso y oportunidades de la transferencia del conocimiento desde la psicología comunitaria a las políticas públicas. Universitas Psychologica, 14(4), 1347-1358. doi: 10.11144/Javeriana.up14-4.potc

Alfaro, J., \& Zambrano, A. (2009). Psicología Comunitaria y Políticas Sociales en Chile. Psicologia \& Sociedade, 21 (2), 275-282.

Berroeta, H. (2011). Apuntes para una Intervención Psicosocial con Incidencia. Castalia, 13 (19), 3750 .

Berroeta, H., Hatibovic, F., \& Asún, D. (2012). Psicología Comunitaria: prácticas en Valparaíso y visión disciplinar de los académicos nacionales. Polis, 11(31), 335-354. doi $: 10.4000 /$ polis.3756

Berroeta, H. (2014). El quehacer de la psicología comunitaria: Coordenadas para una cartografía. Psicoperspectivas, 14(2), 19-31. doi: 10.5027/PSICOPERSPECTIVAS-VOL13-ISSUE1FULLTEXT-352

Bishop, B. J., Vicary, D. A., Browne, A. L., \& Guard, N. (2009). Public policy, participation and the third position: The implication of engaging communities on their own terms. American Journal of Community Psychology, 43(1-2), 111-121. doi: 10.1007/s10464-008-9214-8

Braun, V., \& Clarke, V. (2006). Using thematic analysis in psychology. Qualitative Research in Psychology, 3, 77-101. doi:10.1191/1478088706qp063oa

Braun, V., \& Clarke, V. (2012) Thematic analysis. En Cooper, H. (Ed.), APA Handbook of Research Methods in Psychology: Vol. 2. Research Designs, (pp. 71-57). doi: 10.1037/13620-004

De la Maza, G. (2004). Políticas públicas y sociedad civil en Chile: el caso de las políticas sociales (1990-2004). Política, 43, 105-148. Recuperado de http://www.redalyc.org/articulo.oa?id=64504306

Krause, M., Jaramillo, A., Monreal, V., Carvacho, H., \& Torres, A. (2011). Historia de la Psicología Comunitaria en Chile: Desde la Clandestinidad a la Política Pública. En M.

Lapalma, A., \& De Lellis, M. (2012). Psicología comunitaria y políticas públicas. Una articulación posible y necesaria. En J. Alfaro, A. Sánchez \& A. Zambrano (Eds.), Psicología comunitaria y políticas sociales: reflexiones y experiencias (pp. 147-172). Buenos Aires, Argentina: Paidós.

Montero, M. (2001). Ética y política en psicología. Las dimensiones no reconocidas. Athenea Digital.

Montero, M. (2004). Introducción a la Psicología Comunitaria. Buenos Aires, Argentina: Paidos.

Montero, M \& Serrano-García, I. (2011). Historias de la psicología comunitaria en América Latina. Participación y Transformación (pp. 115-138). Buenos Aires, Argentina: Paidós 
Nelson, G., Lavoie, F., \& Mitchell, T. (2007). The history and theories of community psychology in Canada. En S. Reich, M. Riemer, I. Prilleltensky \& M. Montero (Eds.), International Community Psychology: History and Theories (pp 13-36). doi: 978-0-387-49500-2_2

Nelson, G. (2013). Community psychology and transformative policy change in the neo-liberal era. American Journal of Community Psychology, 52(3), 211-223. doi: 10.1007/s10464-0139591-5

Perkins, D. (1995). Speaking truth to power: empowerment ideology as social intervention and policy. American Journal of Community Psychology, 23(5), 730-765.

Phillips, D. (2000). Social policy and community psychology. En J. Rappaport \& E. Seidman (Eds.), Handbook of community psychology (pp. 397-420). doi: 10.1007/978-1-4615-4193-6

Prilleltensky, I. (2004). En Montero, M. Introducción a la Psicología Comunitaria. Buenos Aires, Argentina: Paidos.

Raczynski, D., \& Serrano, C. (2005). Las políticas y estrategias de desarrollo social: aportes de los años 90 y desafíos futuros. En Meller, P. (Ed.), La paradoja aparente. Equidad y Eficiencia: Resolviendo el dilema (pp. 225-283). Santiago de Chile: Taurus.

Reyes, M. (2007). Orientaciones ético-valóricas de la Psicología Comunitaria en Chile: Análisis descriptivo de los artículos publicados en revistas nacionales entre 1993 y 2003 . En J. Alfaro \& H. Berroeta (eds). Trayectoria de la Psicología Comunitaria en Chile (pp. 109-141).

Reyes, M. I., Olivares, B., Berroeta, H., \& Winkler, M. I. (2015). Del discurso a las prácticas:

Políticas sociales y psicología comunitaria en Chile. Polis, (42). Recuperado de

http://polis.revues.org/11413

Rodríguez, A. (2009). Social policies in Uruguay: A view from the political dimension of Community Psychology, American Journal of Community Psychology, 43(1), 122-133. doi: 10.1007/s10464008-9213-9

Rodríguez, A. (2012). Aportes de la Psicología Comunitaria al campo de las políticas públicas sociales: el caso del Uruguay. En J. Alfaro, A. Sánchez \& A. Zambrano (Eds.), Psicología Comunitaria y Políticas Sociales: reflexiones y experiencias (pp. 111-146). Buenos Aires, Argentina: Paidós.

Ruiz, J. (2012). Metodología de la investigación cualitativa. Bilbao, España. Bilbao Universidad de Deusto.

Serrano-García, I. (2013). Social policy: The tightwire we walk (a commentary). Global Journal of Community Psychology Practice, 4(2). Recuperado de http://www.gjcpp.org/

Shinn, M. (2007). Waltzing with a monster: Bringing research to bear on public policy. Journal of Social Issues, 63(1), 215-231. doi: 10.1111/j.1540-4560.2007.00505.x

Willig, C. (2013). Introducing Qualitative Research In Psychology (3ra Edición). Nueva York, Estados Unidos de América: McGraw-Hill / Open University Press.

Winkler, M. I., Alvear, K., Olivares, B., \& Pasmanik, D. (2012). Querer no basta: Deberes éticos en la práctica, formación e investigación en psicología comunitaria. Psykhe, 21(1), 115-129.

Winkler, M., Pasmanik, D. Alvear, K., \& Olivares, B. (2015). La dimensión ética en Psicología Comunitaria: Orientaciones específicas para la práctica y la formación. Santiago, Chile: Universidad de Santiago de Chile.

Zambrano, A., \& Bustamante, G. (2012). La Política Social en el Espacio Local, ¿Puede Aportar al Desarrollo Comunitario? Un análisis en la Región de la Araucanía, Chile. En J. Alfaro, A. Sánchez \& A. Zambrano (Eds.), Psicología Comunitaria y Políticas Sociales: Reflexiones y Experiencias (pp. 333- 355). Buenos Aires, Argentina: Paidós. 